\title{
Spatial variation of the pickup-proton-injection rate into the ACR regime at the 3D-heliospheric termination shock
}

\author{
K. Scherer ${ }^{1}$ and H.-J. Fahr ${ }^{2}$ \\ 1 Institut für Theoretische Physik IV, Ruhr Universität Bochum, 44780 Bochum, Germany \\ e-mail: kls@tp4.rub.de \\ 2 Argelander Institut für Astronomie, Universität Bonn, Auf dem Hügel 71, 53121 Bonn, Germany \\ e-mail: hfahr@astro.uni-bonn.de
}

Received 11 August 2008 / Accepted 18 December 2008

\begin{abstract}
Context. Anomalous cosmic rays are thought to be generated from pick-up ions that undergo Fermi-1 acceleration in the region close to the solar wind termination shock. This selective acceleration process depends on the efficiency at which upstream pick-up ions are injected into the first reflection mode, from where acceleration into higher energy anomalous cosmic rays starts.

Aims. The injection efficiency is highly sensitive to the local inclination of the upstream magnetic field with respect to the shock normal. We determine the probability of pick-up ions being reflected at the shock depending on the magnetic inclination angle, which varies characteristically with latitude and longitude. This reflection is the relevant initial step in the acceleration of pickup ions to anomalous cosmic rays.

Methods. We point out that the most dramatic change in the magnetic tilt angle occurs whenever two consecutive solar wind sector structures with different magnetic polarities, pass over the shock. We treat this effect by modeling the 3D- variation of the injection efficiency and start from calculations of the pick up ion reflection probability as function of the magnetic tilt angle. We first derive the magnetic tilt angle as function of latitude and longitude, representing the 3D-shape of the termination shock by a triaxial or prolate ellipsoid adaptable for conditions of different solar cycles, respectively. This allows us to semi-analytically express the 3D-variation of the injection efficiency and rate over a solar cycle.

Results. We find a substantial variation in the injection efficiency by 5 orders of magnitude when passing from the upwind parts to the flanks of the termination shock or from low to high latitudes (except at the poles). Folding this with the normalized pickup ion density gives a normalized injection rate which increases from $0 \%$ to $30 \%$ from upwind to crosswind directions and analogously decreases from crosswind into downwind direction. The maximal value of the injection rate highly depends on the underlying sector structure of the heliospheric magnetic field. During solar minimum there is almost no injection in high latitudes, except at the pole itself, while the injection rate is everywhere fairly good during solar maximum.

Conclusions. Our model indicates that the injection into the anomalous cosmic ray acceleration mainly takes place in crosswind directions e.g. in the flanks of the termination shock.
\end{abstract}

Key words. shock waves - Sun: solar wind - magnetohydrodynamics (MHD) - plasmas

\section{Introduction}

Neutral interstellar hydrogen passes over the interface structure of the heliosphere and enters the inner heliosphere, i.e. the supersonic solar wind regime. Upon ionization, they are converted into newly born ions, called pickup ions (PUIs), which are convected outward with the solar wind by the electro-motoric action of frozen-in magnetic fields. While they are forced to comove with the solar wind, they are subject to processes like adiabatic cooling and momentum diffusion, and thus need to be described by an appropriate PUI phase-space transport equation.

A solution of this transport equation was given by Chalov \& Fahr (1996, 2000). Most of the PUIs are swept over the termination shock (TS) and then comove with the subsonic solar wind further downstream. Nevertheless, due to their special conditions in phase space, a small fraction of them are reflected in upstream directions and start undergoing the Fermi-1 acceleration process finally leading to anomalous cosmic rays (ACRs). These special conditions are caused by "adiabatic reflections" (i.e. magnetic mirroring) in the condensed (downstream) magnetic fields:, i.e., they are connected with low ion velocities relative to the shock, which are represented by ions sitting in the sunward hemisphere of the shell-like distribution in the upstream wind frame.

After the first reflection, pitch-angle scattering brings the reflected ions into an extended velocity shell of the ion population comoving with the upstream solar wind. This behavior of reflected PUIs is described by the above mentioned authors as a PUI reflectance efficiency, which is very sensitive to the magnetic tilt angle $\psi$ between the magnetic field and the shock normal. To avoid confusion with the tilt angle of the heliospheric current sheet, we herewith refer to the angle between the magnetic field vector and the normal of the termination shock surface, as the "magnetic tilt angle".

In the following we apply the results of these authors (Fahr et al. 2008) to a 3D-shock geometry and develop a 3D-efficiency that depends on both heliospheric latitude and longitude of the TS. For that we represent the 3D-shape of the TS by the surface of a tri-axial ellipsoid, with one focus at the Sun and with upwind and downwind locations adapted to model calculations by Borrmann \& Fichtner (2005). The magnetic field in the inner heliosphere upstream of the TS is calculated using the Parker field, allowing for an analytic representation of the 

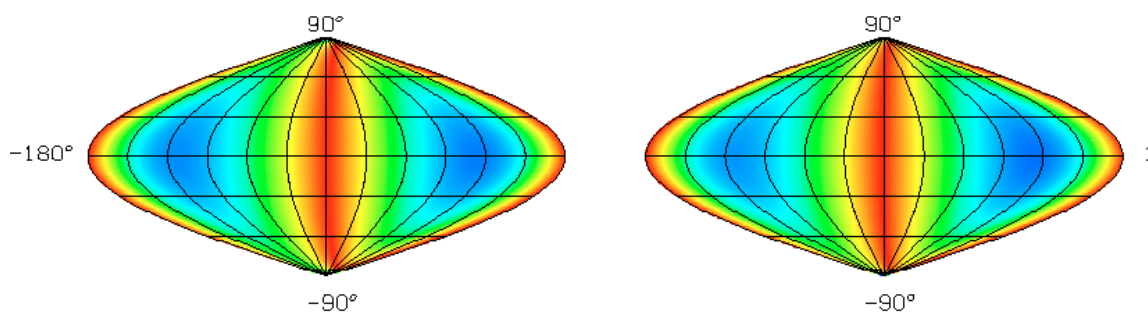

$180^{\circ}$
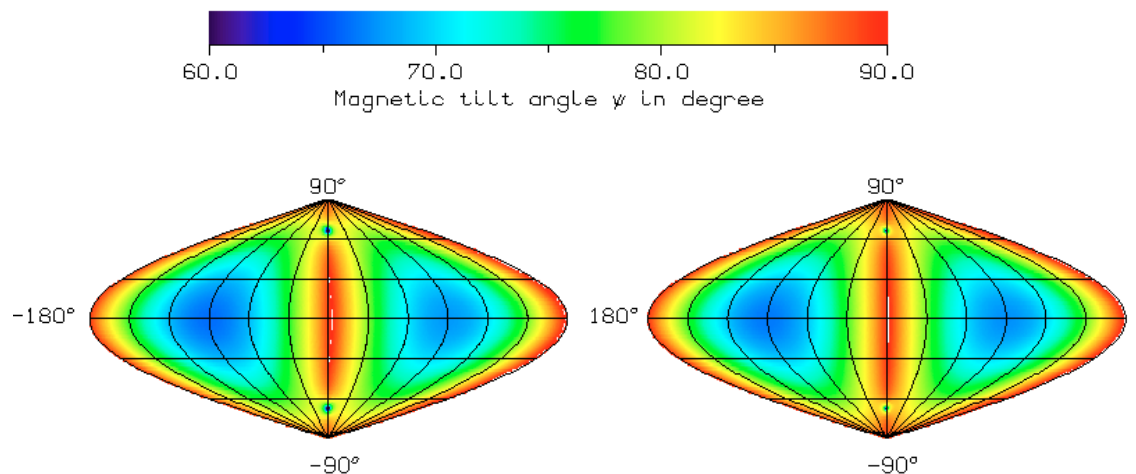

180

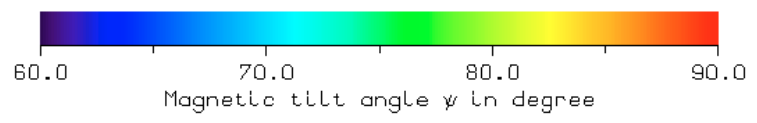

Fig. 1. The magnetic tilt angle at the termination shock in an elliptic projection in heliocentric coordinates $(\varphi, \vartheta)$ is presented. The solar minimum conditions are shown in the left panel, while that during solar maximum are shown in the right panel. For the minimum condition a triaxial ellipsoid is used to describe the position of the termination shock, where for latitudes higher $\pm 35^{\circ}$ a high speed solar wind is taken into account. For solar minimum an prolate ellipsoid $(b=c)$ represents the structure of the termination shock, because the low speed solar wind fills the entire heliosphere. For a further discussion see text.

Fig. 2. Same as Fig. 1 in eccentric anomalies $(E, F)$. The singularity at the pole in heliocentric coordinates is now projected to the point $E=0^{\circ}, F \approx \pm 75^{\circ}$. Here the differences bewteen solar minimum and maximum become more evident, at the poles, where during solar maximum (right panel) the magnetic tilt angle decreases faster for $|F|>\approx 75^{\circ}$ compared to that at solar minimum (left panel). magnetic tilt angle $\psi$ against latitude $\vartheta$ and longitude $\varphi$. A complication has also to be taken into account, which is the passage of the heliospheric magnetic field sector over the TS. While a sector structure passes over the TS, the magnetic field vector rotates by about 180 degrees, which strongly influences the magnetic tilt angle $\psi$ and therefore the injection efficiency $E=E(\psi)$ (see Fahr et al. 2008). Respecting the results of Chalov \& Fahr (1996), we are finally able to derive an analytic expression for the 3D- injection efficiency and, connected with it, the injection rate.

In Sect. 1 we introduce a triaxial ellipsoid as an analytic representation of the termination shock and construct the local magnetic tilt angle $\psi$ using Parker's representation of the interplanetary magnetic field. Analytic details enabling a switch from a heliocentric coordinate system $(r, \vartheta, \varphi)$ to a system with a midpoint representation of an ellipsoid $(r, F, E)$ are given in Appendix A. In Sect. 2 we study, with the help of the analytic representation of the magnetic tilt angle $\psi$, the three-dimensional variation of the injection efficiency $E(\psi(\vartheta, \varphi))$, which is the fraction of pick-up ions reflected back into the upstream direction compared to all PUI's running from upstream into the shock.

\section{Analytic representation of the magnetic tilt angle}

\subsection{Representation of the heliosphere as $3 D$-ellipsoid}

A Cartesian coordinate system is chosen so that its $x$-axis points to the inflowing interstellar wind direction, the $z$-axis is parallel to the direction into the solar north pole (ecliptic north pole), and the $y$-axis completes the right-handed coordinate system: a space vector $r$ to a point at a triaxial ellipsoid $(a>c>b)$ is given by:

$\boldsymbol{r}=\left(\begin{array}{l}a \cos E \cos F \\ b \sin E \cos F \\ c \sin F\end{array}\right)$

where, according to the notation used in Celestial Mechanics, $E \in[-\pi, \pi]$ is the eccentric anomaly in the $x-y$ plane, and
$F \in[-\pi / 2, \pi / 2]$ that one in the $x-z$-plane, or the usual latitudinal and longitudinal angles in spherical coordinates $(r, E, F)$. The angles $\varphi, \vartheta$ are the corresponding true anomalies, or the longitude and latitude in heliocentric coordinates $\left(r_{\star}, \varphi, \vartheta\right)$. That means e.g., $F=0, E=0$ corresponds to $x$-axis (semi-major axis $a$ ), $E=0, F=90$ to the $z$-axis (semi-minor axis $c$ ), and $E=90, F=0$ to the $y$-axis (semi-minor axis $b$ ). Because of the above made choice of the coordinate system, the general description of a triaxial ellipsoid with: $a>b>c$ does not hold here, because during solar minimum high speed streams at high latitudes push the termination shock over the solar poles to larger distances ( $z$-axis or semi-minor axis $c$ ) than that in the ecliptical crosswind direction ( $y$-direction or semi-minor axis $b$ ), therefore $c>b$. During solar maximum the entire heliosphere is filled with slow speed solar wind and therefore ecliptical crosswind and polar distances tends to be equal and hence both semi-minor axes are equal, e.g. $b=c$. In this case the ellipsoid is called prolate. Actually, we just need $a>b$ and $a>c$. Throughout the paper the intersection of the line through the solar poles with the termination shock are denoted "heliospheric poles", which are the "poles" $\left(\vartheta=90^{\circ}\right)$ in the heliospheric representation (Fig. 1) or the points at $E=0^{\circ}, F \approx \pm 75^{\circ}$ in the midpoint representation (Fig. 2 and following).

Modeling the time-dependent heliosphere in three dimensions requires an adequate modeling of solar cycle related high speed streams in the heliosphere. This modeling includes the interaction of interstellar protons and neutral gases with the supersonic solar wind protons (e.g. Borrmann \& Fichtner 2005) and involved magnetic fields (Pogorelov et al. 2006; Opher et al. 2006), and also includes cosmic rays (Fahr et al. 2000; Ferreira et al. 2007), and time-dependent dynamical effects of the solar activity cycle (Scherer \& Ferreira 2005; Izmodenov et al. 2008). Here we do not go into that discussion, but refer the reader to Müller et al. (2008), where some of these models are compared. We just note, that during solar maximum condition the entire volume of the heliosphere is filled with low speed solar wind, 
while during solar minimum conditions a high speed solar wind evolves over the solar poles, e.g. above/below $\pm 35^{\circ}$ from the ecliptic, and persists over a period of about 3 years as observed by Ulysses spacecraft (see McComas et al. 2003).

The magnetic field in the heliosphere is a degenerated dipole field of the sun, with a radial component decreasing like $r^{-2}$, and a latitude-dependent azimuthal component decreasing with $r^{-1}$ vanishing with $\cos \vartheta$ over the heliospheric poles, (e.g. Parker 1958), see also Forsyth et al. (2002) for observational data. This heliospheric magnetic field is given by:

$\boldsymbol{B}=\frac{b_{0}}{r^{2}}\left(\boldsymbol{e}_{r}, 0, \boldsymbol{e}_{\varphi} \frac{\Omega}{v_{\mathrm{sw}}} r \cos \vartheta\right)$

with $b_{0}=B_{0} r_{\odot}^{2}$ and $k \equiv \frac{\Omega}{v_{\mathrm{sw}}}=1.08025 \mathrm{AU}^{-1}$. In the high speed stream also the magnetic spiral field $\boldsymbol{B}$ changes accordingly because of its velocity-dependence: the low speed solar wind typically has $v_{\mathrm{sw}}=400 \mathrm{~km} \mathrm{~s}^{-1}$, while the high speed wind in polar regions (e.g. in latitudes above/below $\vartheta_{\text {high }} \pm 35^{\circ}$ ) has twice that value (e.g. $800 \mathrm{~km} \mathrm{~s}^{-1}$ ). Thus during solar minimum conditions the factor $k$ in high latitudes has to be reduced by a factor 2 . To avoid an unsteady sharp transition at $\pm 35^{\circ}$ we introduce the following function $f(\vartheta)$ :

$f(\vartheta)=0.5\left(\tanh \left(\Delta\left(\vartheta+\vartheta_{0}\right)\right)-\tanh \left(\Delta\left(\vartheta-\vartheta_{0}\right)\right)\right)$

with $\Delta=10$ and $\vartheta_{0}=35$ which allows a smooth transition between high speed and low speed solar wind regions. The heliocentric coordinate for the high speed boundary $\vartheta_{\text {high }}= \pm 35^{\circ}$ translates to $F_{\text {high }}= \pm 26^{\circ}$ eccentric latitude. In the figures described below this values is to close to the $F=30^{\circ}$ grid line to become resolved. Therfore, we did not plot it into our elliptical projections.

According to the model by Borrmann \& Fichtner (2005) the distance of the termination shock in heliocentric coordinates $(r, \varphi, \vartheta)$ given in Scherer et al. (2006b): $r_{\text {nose }}=85 \mathrm{AU}, r_{\text {pole }}=$ $130 \mathrm{AU}, r_{\text {tail }}=189 \mathrm{AU}$. With the perihelion distance $p=a(1-\epsilon)$ and the aphelion distance $q=a(1+\epsilon)$ it is easy to calculate the following properties of the ellipsoid; $a=(p+q) / 2=137 \mathrm{AU}$, $\epsilon=q / a-1=0.38$, and $b=a \sqrt{\left(1-\epsilon^{2}\right)}=126$ AU. The determination of $c$ follows from the heliocentric polar distance $r=$ $a\left(1-\epsilon_{2}^{2}\right) /\left(1+\epsilon_{2} \cos \varphi\right)$ with $\varphi=90$, which gives with the above values: $r=a\left(1-\epsilon_{2}^{2}\right)$ or $\epsilon_{2}=\sqrt{1-r / a}=\sqrt{1-130 / 137}=0.23$, and $c=a \sqrt{1-\epsilon_{2}}=133 \mathrm{AU}$.

Thus $a=137, b=129, c=133 \mathrm{AU}, \epsilon_{1}=0.38, \epsilon_{2}=0.23$.

Recent Voyager observations (Richardson et al. 2008) suggest the termination shock may have a small tilt to the inflow direction and is blunt. Nevertheless, none of the heliospheric models ever predicted a spherical termination shock, but those which had almost an ellipsoidal character. The tilt angle reported by the cited authors is small and caused either by temporal effect or by a large deviation of the interstellar magnetic field vector from the inflow direction. The latter mainly affects the shape of the heliopause and that of the bowshock, but only weakly that of the termination shock (Pogorelov et al. 2008). Therefore, one can simply rotate the entire ellipsoid, described above, so that its nose points into the indicated symmetry direction. Therefore, the results described below will only be affected weakly by this tilt and are not quantitatively discussed here.

The approximation of the termination shock shape by ellipsoids can be refined using numerical models (for references see above), but here we are interested only in the principal effects. Moreover, the curvature of the modeled termination shock surface should not deviate too much from that of an ellipsoid. The fit to an ellipsoid is worst in the tail region, but there are also the PUI fluxes low. In the nose and heliospheric pole regions the approximation is fairly good as shown in Fahr et al. (2008).

\subsection{Determination of the magnetic tilt angle}

The magnetic tilt angle $\psi$ is given by:

$\psi=\operatorname{arcos}\left(\frac{\boldsymbol{n}_{r} \cdot \boldsymbol{B}}{n_{r} B}\right)$.

Details of the explicit calculation can be found in Appendix A and $\mathrm{B}$.

Since the magnetic tilt angle does not depend on the orientation of the magnetic field or normal vector, we can safely neglect polarity changes between a south or north polar field. Therefore magnetic tilt angles larger than $90^{\circ}$ are simply reduced to $180^{\circ}-\psi$.

The results concerning magnetic tilt angles are presented in Figs. 1 and 2: the solar minimum conditions are shown in the left panel, while solar maximum conditions are presented in the right panel. For the minimum condition the above described triaxial ellipsoid is used to describe the position of the termination shock. In addition, for latitudes higher than $\pm 35^{\circ}$ the high speed solar wind due to less winding causes a stronger decrease of the magnetic field strength. For solar minimum a prolate ellipsoid ( $b=c$ ) represents the structure of the termination shock, because the low speed solar wind prevails in the entire heliosphere. The magnetic tilt angles at the termination shock are given in an elliptical Mercator-Sanson projection with respect to the heliocentric $(\varphi, \vartheta)$ and midpoint $(E, F)$ representation of an ellipsoid (see Figs. 1 and 2, respectively). While for the heliocentric coordinates the differences between solar minimum and maximum vanish in the projection around the poles, the choice of eccentric anomalies projects the heliocentric pole to $E=0^{\circ}, F \approx \pm 75^{\circ}$ eccentric latitude. Thus the midpoint representation makes the differences around the poles visible.

It can be seen in Fig. 2 that no large differences exist between solar minimum and solar maximum conditions. The polar region of the prolate ellipsoid (solar maximum, $b=c$ ) shows a more perpendicular shock (e.g. a nearly $90^{\circ}$ magnetic tilt angle), while during solar minimum $(c>b)$ the polar region are farther away, and a lower magnetic tilt angle can be observed at high latitudes $\pm F$. In both parts of the solar activity cycle the magnetic tilt angle is low in the flanks, thus the magnetic field configuration resembles more a quasi-parallel, or oblique shock. Following in Fig. 2 the line at an eccentric latitude $\vartheta=0^{\circ}$ reflects the result of our two-dimensional approach (Fahr et al. 2008), which also showed an oblique shock configuration in the ecliptic. In the next section we use the above result to study the correlated injection efficiency and rate.

\subsection{Efficiency and rate of injection}

The injection efficiency describes the fraction of pick-up ions, amongst all swept out to the termination shock, which are injected into an acceleration process to higher energies by firstorder reflection to finally become anomalous cosmic rays. An interesting side aspect is, that, depending on injection rates, more or less PUI's undergo the ACR acceleration process and therefore are lost for the production of energetic neutral atoms via charge exchange (PUI-ENAs) (e.g. Fahr \& Scherer 2004; Sternal et al. 2008; Heerikhuisen et al. 2008; Wurz et al. to appear). 

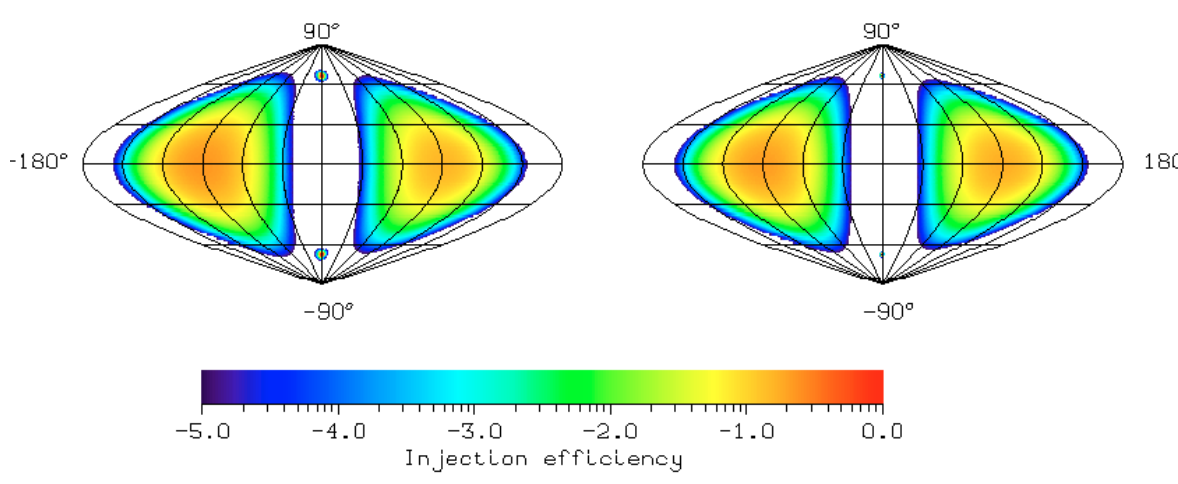

Fig. 3. The right panel shows the injection efficiency at the termination shock. The values are cut below $10^{-5}$. For more detail see Fig. 2 or text.
This latter effect is important for the upcoming IBEX mission (McComas et al. 2000).

In Fahr et al. (2008) an analytic representation of the injection efficiency dependent on the magnetic tilt angle is developed yielding

$$
E(\psi)=E_{0} \exp \left(-k \psi^{n}\right)
$$

which is based on the approach by Chalov \& Fahr (1996), in which the authors assumed average MHD turbulence levels, i.e. with $\left\langle\delta B^{2} / B^{2}\right\rangle=0.1$ (where $\delta B$ is the magnetic field fluctuation) and used a test particle approximation. From this approximation the best fit parameters $k=2.08 \times 10^{-20}$ and $n=10.9$ are derived, where $\psi$ is given in degrees. For more details see Fahr et al. (2008). In Fig. 3 we show the results from inserting Eqs. (4) into (5) to determine the injection rate. As can be seen from that figure the injection rate over the poles almost vanishes in accordance with Eq. (5). The injection efficiency is highest in the flanks, which will allow for higher production rates of anomalous cosmic rays and lower PUI-ENA production rates.

As discussed in Fahr et al. (2008) a sector structure persists approximately between 2 and 10 days, according to Voyager data (Burlaga et al. 2003) and that the polarity change lasts at least one day as suggested by the data points during that time in Burlaga et al. (2003). In Kucharek \& Scholer (1995) it was already discussed that the magnetic tilt angle varies from $0^{\circ}$ to $180^{\circ}$ when the magnetic polarity changes between two consecutive sectors. The period for which one polarity of a sector lasts, is called the persistence time $T$, while the change to the other polarity occurs in a period $\Delta T$ of one day. Assuming a uniform rotation of the magnetic field vector during this day, the magnetic tilt angles $<70^{\circ}$ persist for $80 \%$ of that day, e.g. about $80 \%$ of that period are favorable to injection. As soon as magnetic tilt angles smaller than $70^{\circ}$ are realized, the resulting injection efficiency is almost constant at $E \simeq E_{0}$. Thus dividing this day of change into an efficient fraction $\tau_{\mathrm{e}}$ and an inefficient one $\tau_{\mathrm{i}}$, including the persistence time $T$, we express the mean injection efficiency $\tilde{E}$ by

$\tilde{E}=\frac{\left(T+\tau_{\mathrm{i}}\right) E(\psi)+\tau_{\mathrm{e}} E_{0}}{T+\tau_{\mathrm{e}}+\tau_{\mathrm{i}}}$.

As described above, $\tau_{\mathrm{e}} \approx 0.8$ and $\tau_{\mathrm{i}} \approx 0.2$.

The injection rate is given by the product of the injection efficiency, the number density of the PUIs and the solar wind $V_{\mathrm{sw}}$ speed at the termination shock. We fit the PUI-flux $\Phi_{\mathrm{PUI}}(\varphi, \vartheta)=n_{\text {up,PUI }}\left(r_{\mathrm{TS}}, \varphi, \vartheta\right) V_{\mathrm{sw}}\left(r_{\mathrm{TS}}, \varphi, \vartheta\right)$, normalized to the flux level at the nose of the TS, by a straight line $\Phi^{\prime}(\varphi, \vartheta)=$ $\left.\Phi_{\mathrm{PUI}}(\varphi, \vartheta) / \Phi_{\mathrm{PUI}}(0,0)=A_{1} \varphi+A_{2} \vartheta+A_{3}\right)$, according to that discussed for solar minimum by Scherer et al. (2006a), with $A_{1}=-3.9 \times 10^{-3}$ and $A_{3}=1$, i.e. $\Phi^{\prime}\left(0^{\circ}\right)=1$ and $\Phi^{\prime}\left(180^{\circ}\right)=0.3$.
Because the model of the latter authors is two-dimensional axial symmetric, and to present knowledge, no three dimensional distribution of PUIs is available in literature, we assume here that the dependence in latitude and longitude for the PUI number density essentially is identical, e.g. $A_{2}=0$. In fact it is smaller over the poles during solar minimum, because due to flux conservation the product $n_{\mathrm{sw}} v_{\mathrm{vsw}}$ is constant (McComas et al. 2003), hence an increasing solar wind speed during that period requires a decreasing solar wind number density. Moreover the cross section of the charge exchange process is velocity-dependent (for a detailed discussion see the review by Fahr et al. 2007). Nevertheless, in our approximation, which is intended to study the principal effects, it is sufficient to use a latitude-independent distribution of PUIs at the termination shock.

In the following we study the normalized injection rates, $\beta$, which is defined as the product of the normalized injection efficiency and the normalized PUI flux.

During solar maximum the solar magnetic dipole appears tilted by almost $80^{\circ}$ (Zhao et al. 2005). This does not effect the overall magnetic field structure at the termination shock, except for the position of the current sheet. The latter is a plane in which the neutral line of the magnetic field is located, and above and below of which almost identical magnetic field magnitudes prevail, but the polarity is different. As discussed above, we safely can neglect polarity effects. Moreover, since the r-component of the field is radially symmetric, e.g. decreases with $r^{-2}$, it is rotationally invariant. The azimuthal component is due to the solar rotation, and therefore the overall field structure at the termination shock is the same during solar maximum and minimum, except for polarity, differential winding caused by high speed streams. The position of the current sheet projected to the shock surface appears as a thin line. Therefore, albeit its importance for cosmic ray transport (Potgieter 2008) we neglect it in the further discussion. Also we neglect transient phenomena like global merging interaction regions (GMIRs), where the magnetic field can have a structure different from the Parker spiral field. During that time preferred injection zones are possible. In our simple approach we also do not take into account propagation effects between nose, pole or tails, which means that the magnetic field configuration we describe, arrive almost instantaneously at the termination shock. This approach is not a problem for long-term averages, but for transient phenomena it is. The latter needs a detailed modeling of a 3-dimensional dynamic heliosphere, which is not our aim here. We rather want to describe by our analytic approach the general physical effects of an average magnetic field configuration.

This is shown in Figs. 4 and 5 for solar minimum and maximum conditions. During solar minimum no sector structure occurs above latitudes of $\pm 35^{\circ}$, and therefore in polar regions the injection rate is almost zero, except near the poles, where by the 


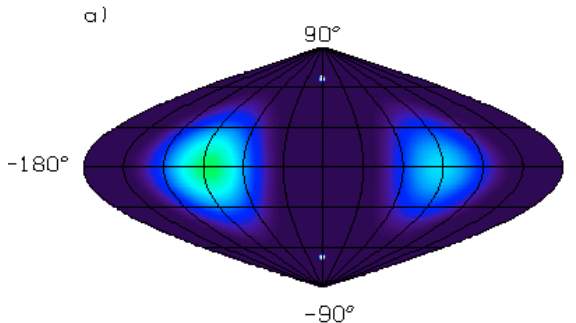

c)
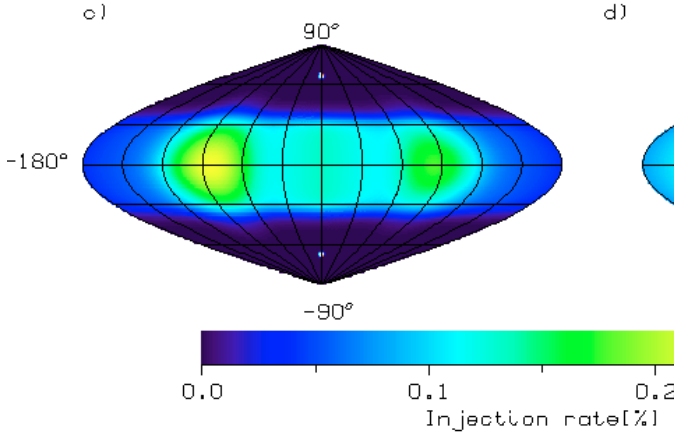

d)
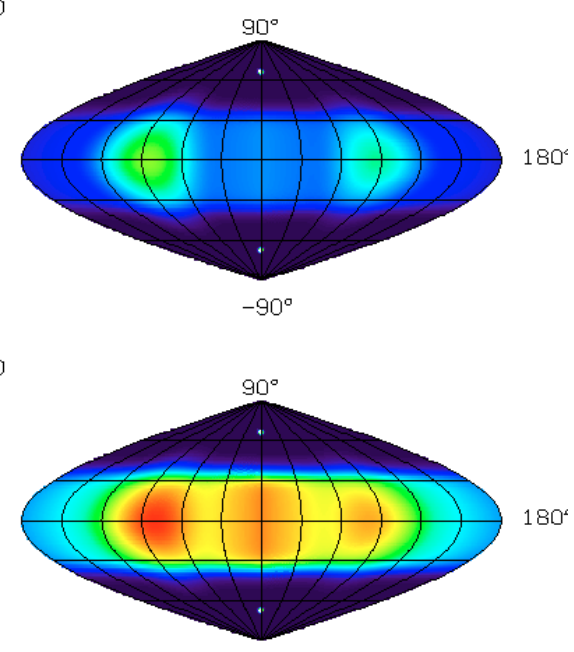

Fig. 4. The injection efficiency for solar minimum conditions at the termination shock. The panels a) to d) model a persistence time of the sector structure for $T=\infty, 10,5,2$ days. For more details see text and Fig. 2.

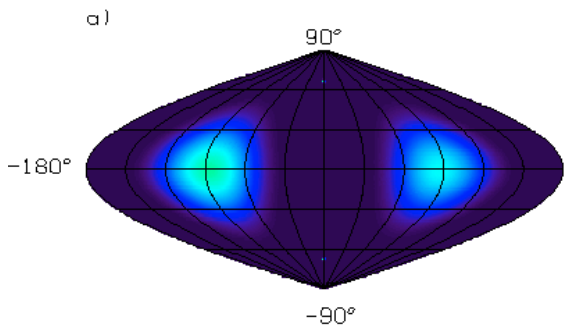

c)

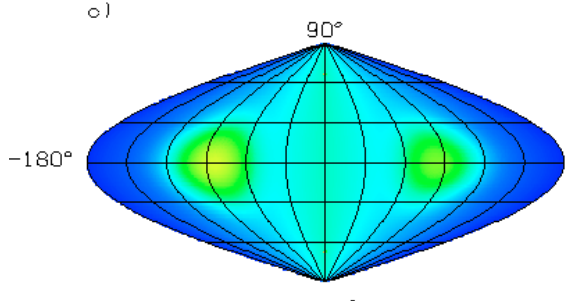

$-90^{\circ}$
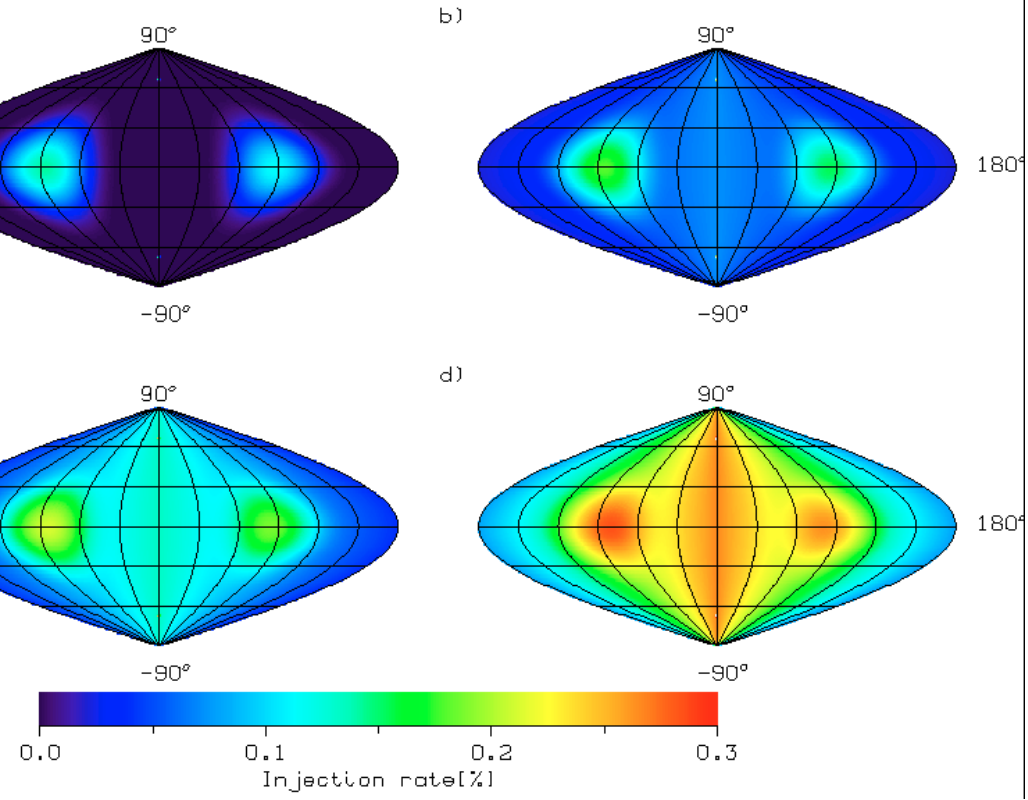

Fig. 5. The injection efficiency for solar maximum conditions at the termination shock. See text and Figs. 2 and 4 for details. physics of the Parker magnetic field is purely radial, and hence the shock normal is almost parallel to the magnetic field vector. In low ecliptic latitudes the sector structure is present. The persistence times of the sector are $T=\infty, 10,5,2$ days corresponding to panels a), b), c), and d), respectively, where the persistence time " $\infty$ " means, that no change of sectors occurs, and, therefore, always the same polarity is present. It can be seen that all of the injection happens in the flanks, with an additional small region around the poles. The effect increases with decreasing persistence times. For the smaller persistence times also a strip around $0^{\circ}$ longitude from pole to pole becomes a preferable injection region.

For solar maximum we have the tilted solar dipole (Zhao et al. 2005) which introduces a sector structure in the entire heliosphere. It can be seen in Fig. 5 that at that times almost everywhere good there are conditions for accelerating particles are, i.e. the injection rate is high everywhere. Again, the highest injection rates are found in the flanks.
The poles remain unaffected during solar minimum and maximum conditions, because of the dipole character of the Parker spiral field: the far field of the dipole is always radial, and the spiral is impressed by solar rotation, for which the winding angle depend only on the latitude, e.g. vanishes over the pole by its nature.

As discussed in our previous letter (Fahr et al. 2008) there exist other attempts to describe the high injection rate at the flanks, e.g. Chalov (1993); Schwadron \& McComas (2003); McComas \& Schwadron (2006) based on qualitative arguments. A blunt termination shock in the tail region exists only in models with a single species, namely protons, while those including neutral hydrogen atoms, smooth it (see Müller et al. 2008, for a comparison of such models). We generalized the (local) findings by Kucharek \& Scholer (1995); Chalov \& Fahr (1996); Chalov et al. (1997); Chalov \& Fahr (2000); Chalov (2005) to a global three-dimensional semi-analytic approach using a simplified ellipsoidal geometry of the termination shock. 


\section{Conclusions}

We constructed a global three-dimensional semi-analytic approach using a simplifying ellipsoidal geometry for the termination shock to calculate the tilt angle between the magnetic field vector and the shock surface normal. With this approach we then could finally determine the injection rate of PUIs into the ACR- acceleration process. Even, if the ellipsoidal surface differs in the tail region from numerical models, its curvature is similar, i.e. our estimated magnetic tilt angle may only slightly vary from that of numerical models. But this does not change the principle physics as outlined below.

From Figs. 4 and 5 we can conclude that the favorable acceleration regions are always the flanks of the 3D-heliosphere. During solar minimum, these regions are further restricted to latitudes below $\vartheta_{\text {high }}= \pm 35^{\circ}$ or $F_{\text {high }}= \pm 26^{\circ}$, e.g. to regions close to the ecliptic. The time variable sector structure creates additional regions, e.g. at the nose, which always are less effective than those in the flanks. During solar maximum conditions the varying sector structure induces at the entire heliospheric termination shock fairly good injection rates. For quite short persistence times ( 2 days) also the nose region becomes as effective as the flanks in injection PUI into the ACR acceleration regime. We did not change the transition time, e.g. the time between two consecutive sectors, because such a study would lead beyond the scope of this paper.

We have demonstrated how variable in latitude, longitude and time the PUI injection into the ACR regime is at the threedimensional termination shock. This result is important with respect to numerically modeling solutions of the ACR phase-space transport equation. Now, for the first time three-dimensional ACR injection sources, as derived in this paper, can be taken into account. It will be interesting what differences in qualitative and quantitative terms the above study will induce into the ACR models published so far.

It should be mentioned, that the above injection study is based on some assumptions on the phase-space transport of PUI's to the distant heliosphere which may have to be changed under the given auspices of more recent PUI transport aspects. For instance, it has recently been revealed that energy diffusion of PUI's in a selfconsistently maintained hydromagnetic turbulence field only leads to weakly pronounced PUI energy tails (Chalov et al. 2004, 2006a,b), somehow putting in question the earlier results of pick-up ion acceleration in co-convected turbulence fields which were used to calculate the PUI injection efficiency (Chalov \& Fahr 1996). If PUI's arrive at the termination shock less pre-accelerated than assumed in this latter paper, then we also have to change the injection rates correspondingly.

On the other hand, this can still be considered as an open problem, since there are claims that PUI's form accelerated tail distributions in the form of power laws (Fisk \& Gloeckler 2006; Gloeckler \& Fisk 2007; Fisk \& Gloeckler 2007; Fahr 2007; Fahr $\&$ Siewert 2008). The question of more or less effective PUI injection then very much depends on the question of how far these power laws extend to higher energies and exist at larger heliocentric distances.

Despite the uncertainty in the PUI injection efficiency, the magnetic tilt angle between a Parker spiral field and a triaxial ellipsoid for the solar minimum conditions and an prolate ellipsoid for solar maximum conditions are uneffected. Because the corresponding magnetic tilt angle is smallest in the flank regions, one would expect always there higher injection due to the more favorable magnetic field configuration.

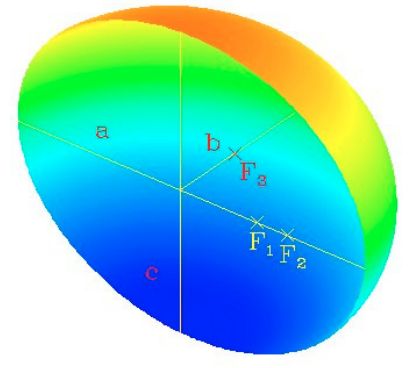

Fig. A.1. A three dimensional representation of a triaxial ellipsoid: $a>$ $b>c$. The foci are denote by $F_{i}$, where $i \epsilon\{1,2,3\}$ and the indices " 1 " describes the $a, b$, "2", the $a, c$ and " 3 " the $b, c$ ellipse, respectively. The $F_{3}$ focus and the corresponding ellipse are not of interest here.

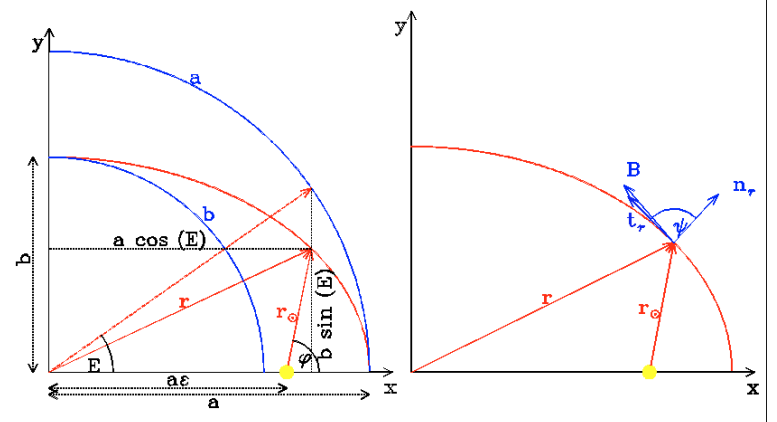

Fig. A.2. Left panel: the transformation from true to eccentric anomalies. Right panel: sketch of the magnetic tilt angle $\psi$, the normal to the surface $\boldsymbol{n}_{r}$, and the magnetic field vector $\boldsymbol{B}$.

The main conclusion that can be drawn from the above considerations is that the favorable acceleration regimes for ACRs are always in the flanks independent of the solar cycle and the underlying sector structure. The changing polarity during a passage of a sector boundary allows for more efficient injection rates in other areas of the termination shock, like at the nose during solar minimum or in the entire heliosphere at solar maximum.

In a forthcoming paper we shall discuss other magnetic field configurations, like the Fisk field (Fisk 1996; Fisk \& Schwadron 2001; Burger et al. 2008) and study its influence on the magnetic tilt angle and finally on the PUI injection at the termination shock.

\section{Appendix A: The normal vector to an triaxial ellipsoid}

We need the representation of the eccentric anomalies in true anomalies and vice versa:

A vector $\boldsymbol{r}$ in the midpoint representation is given by:

$\boldsymbol{r}=\left(\begin{array}{l}a \cos E \cos F \\ b \sin E \cos F \\ c \sin F\end{array}\right)$

while that in heliocentric coordinates $\boldsymbol{r}_{\star}$ is (see right panel of Fig. A.2);

$\boldsymbol{r}=\boldsymbol{r}_{\star}+\left(\begin{array}{l}a \epsilon_{1} \\ 0 \\ 0\end{array}\right)$

With the standard representation of an ellipse $(F=\vartheta=0)$, e.g. $r_{\star}=a\left(1-\epsilon_{1}^{2}\right) /\left(1+\epsilon_{1} \cos \varphi\right)$ one can transform between the 
midpoint (eccentric anomalies) and heliocentric (true anomalies) coordinates:

$\cos E=\frac{\epsilon_{1}+\cos \varphi}{1+\epsilon_{1} \cos \varphi} \quad \sin E=\sqrt{1-\epsilon_{1}^{2}} \frac{\sin \varphi}{1+\epsilon_{1} \cos \varphi}$

or vice versa:

$\cos \varphi=\frac{\cos E-\epsilon_{1}}{1-\epsilon_{!} \cos E} \quad \sin \varphi=\sqrt{1-\epsilon_{1}^{2}} \frac{\sin E}{1-\epsilon_{1} \cos E}$.

The same holds true for the latitude midpoint coordinates $F$ and the heliospheric coordinate $\vartheta$ (e.g. $E=\varphi=0$ ), see Fig. A.3:

$\boldsymbol{r}=\boldsymbol{r}_{\star}+\left(\begin{array}{l}a \epsilon_{2} \\ 0 \\ 0\end{array}\right) \quad$ with $\quad \boldsymbol{r}_{\mathrm{s}}=\boldsymbol{r}_{\star}-\left(\begin{array}{l}a \epsilon_{2}-a \epsilon_{1} \\ 0 \\ 0\end{array}\right)$

follows again:

$\boldsymbol{r}=\boldsymbol{r}_{\star}+\left(\begin{array}{l}a \epsilon_{1} \\ 0 \\ 0\end{array}\right)$

The norm of the heliocentric vector $r_{\star}$ is

$r_{\star}=$

$\sqrt{c^{2} \sin ^{2} F+b^{2} \sin ^{2} E \cos ^{2} F+a^{2}\left(\cos E \cos F-\epsilon_{1}\right)^{2}}$.

Now we construct the tangential vectors to a triaxial ellipsoid:

$\boldsymbol{t}_{E}=\frac{\partial \boldsymbol{r}}{\partial E}=\left(\begin{array}{l}-a \sin E \cos F \\ b \cos E \cos F \\ 0\end{array}\right)$

and

$\boldsymbol{t}_{F}=\frac{\partial \boldsymbol{r}}{\partial F}=\left(\begin{array}{l}-a \cos E \sin F \\ -b \sin E \sin F \\ c \cos F\end{array}\right)$

and then

$$
\begin{aligned}
\boldsymbol{n}_{r} & =\frac{\boldsymbol{t}_{E} \wedge \boldsymbol{t}_{F}}{\left|\boldsymbol{t}_{E} \wedge \boldsymbol{t}_{F}\right|} \\
& =\frac{1}{\left|\boldsymbol{t}_{E} \wedge \boldsymbol{t}_{F}\right|}\left(\begin{array}{l}
b c \cos E \cos ^{2} F \\
a c \sin E \cos ^{2} F \\
a b \sin F \cos F
\end{array}\right),
\end{aligned}
$$

with:

$$
\begin{aligned}
\left|\boldsymbol{t}_{E} \wedge \boldsymbol{t}_{F}\right|^{2}= & {\left[a^{2} b^{2} \sin ^{2} F\right.} \\
& \left.+\left(a^{2} c^{2} \sin ^{2} E+b^{2} c^{2} \cos ^{2} E\right) \cos ^{2} F\right] \cos ^{2} F \\
= & n_{r}^{2} \cos ^{2} F
\end{aligned}
$$

and the normal vector to the ellipsoid in heliospherical coordinates is:

$\boldsymbol{n}_{r}=\frac{1}{n_{r}}\left(\begin{array}{l}b c \cos E \cos F \\ a c \sin E \cos F \\ a b \sin F\end{array}\right)$

Because $\cos F$ is always positive, last equation is independent of the direction of $\cos F$.

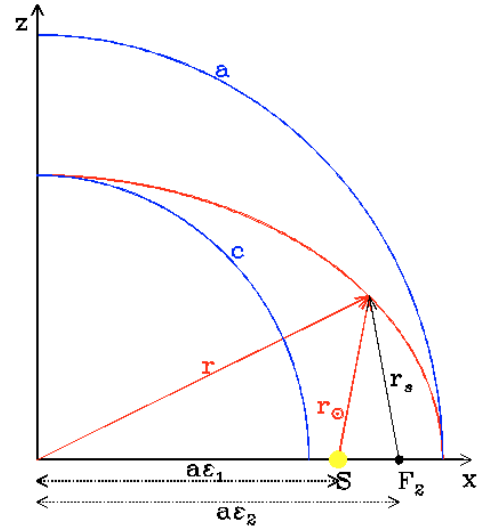

Fig. A.3. The configuration in the $x-z$ plane. $F_{c}$ denotes the focus of the $a, c$-ellipse, while $S$ is the focus of the $a, b$-ellipse, where the Sun is located. $\boldsymbol{r}_{\mathrm{s}}$ is the vector from the focus $F_{c}$ to the required point at the ellipsoid.

\section{Appendix B: The Parker spiral field in heliocentric coordinates}

The Parker spiral field in Cartesian sun-centered coordinates (heliocentric polar coordinates) is:

$$
\begin{aligned}
\boldsymbol{B} & =\frac{b_{0}}{r_{\star}^{2}}\left(\boldsymbol{e}_{r}, 0, k r_{\star} \cos \vartheta \boldsymbol{e}_{\varphi}\right) \\
& =\frac{b_{0}}{r_{\star}^{2}}\left(\begin{array}{l}
\cos \varphi \cos \vartheta \\
\sin \varphi \cos \vartheta \\
\sin \vartheta
\end{array}\right)+\frac{b_{0} k}{r_{\star}}\left(\begin{array}{l}
-\sin \varphi \cos \vartheta \\
\cos \varphi \cos \vartheta \\
0
\end{array}\right)
\end{aligned}
$$

with $b_{0}=B_{0} r_{0}^{2}$ and $k=\frac{\Omega}{v_{\mathrm{sw}}}=1.08025 \mathrm{AU}^{-1}$, and $r_{\star}$ is the radius vector in heliocentric coordinates. Note: The usual $\sin \vartheta$ in the $\vartheta$-component is replaced by $\cos \vartheta$ because of the choice of the coordinate system, see above.

The norm of the magnetic field $|B|$ is easily read of from Eq. (B.1):

$|B|=\frac{b_{0}}{r_{\star}^{2}} \sqrt{1+k^{2} r_{\star}^{2} \cos ^{2} \vartheta}=n_{B} \frac{b_{0}}{r_{\star}^{2}}$.

Inserting all the dependencies Eqs. (A.9) in Eqs. (A.12) and (B.1) lead to clumsy expressions, when calculating the magnetic tilt angle $\psi$ :

$$
\begin{aligned}
\cos \psi=\frac{1}{n_{r} n_{B}} & \left(\begin{array}{l}
b c \cos E \cos F \\
a c \sin E \cos F \\
a b \sin F
\end{array}\right) \cdot\left[\left(\begin{array}{l}
\cos \varphi \cos \vartheta \\
\sin \varphi \cos \vartheta \\
\sin \vartheta
\end{array}\right)\right. \\
& \left.+\frac{b_{0} k}{r_{\star}}\left(\begin{array}{l}
-\sin \varphi \cos \vartheta \\
\cos \varphi \cos \vartheta \\
0
\end{array}\right)\right] .
\end{aligned}
$$

All the transformation from the heliocentric into the midpoint system are given in Eqs. (A.3) and (A.4) and a further analytic calculation will not be performed, because this leads to clumsy expression. Nevertheless, the coordinate transformations between the two systems using Eqs. (A.3), (A.4) and (B.3) can easily implemented into a computer code. Note: there is an ambiguity in the above transformation from the midpoint to the heliospheric coordinate system (and vice versa). To overcome this, we calculated the angle between the heliospheric radius vector $r_{\star}$ and the $z$-axis to get the angle $\vartheta$. 


\section{References}

Borrmann, T., \& Fichtner, H. 2005, Adv. Space Res., 35, 2091

Burger, R. A., Krüger, T. P. J., Hitge, M., \& Engelbrecht, N. E. 2008, ApJ, 674, 511

Burlaga, L. F., Ness, N. F., \& Richardson, J. D. 2003, J. Geophys. Res. (Space Phys.), 108, 8028

Chalov, S. V. 1993, Planet. Space Sci., 41, 133

Chalov, S. V. 2005, Adv. Space Res., 35, 2106

Chalov, S. V., \& Fahr, H. J. 1996, Sol. Phys., 168, 389

Chalov, S. V., \& Fahr, H. J. 2000, A\&A, 360, 381

Chalov, S. V., Fahr, H. J., \& Izmodenov, V. 1997, A\&A, 320, 659

Chalov, S. V., Alexashov, D. B., \& Fahr, H. J. 2004, A\&A, 416, L31

Chalov, S. V., Alexashov, D. B., \& Fahr, H.-J. 2006a, ApJS Trans., 2, 19

Chalov, S. V., Alexashov, D. B., \& Fahr, H. J. 2006b, Astron. Lett., 32, 206

Fahr, H. J. 2007, Annales Geophysicae, 25, 2649

Fahr, H.-J. \& Scherer, K. 2004, Ap\&SS Trans., 1, 3

Fahr, H.-J., \& Siewert, M. 2008, A\&A, 484, L1

Fahr, H. J., Kausch, T., \& Scherer, H. 2000, A\&A, 357, 268

Fahr, H.-J., Fichtner, H., \& Scherer, K. 2007, Rev. Geophys., 45, 4003

Fahr, H. J., Scherer, K., Potgieter, M. S., \& Ferreira, S. E. S. 2008, A\&A, 486, L1

Ferreira, S. E. S., Potgieter, M. S., \& Scherer, K. 2007, ApJ, 659, 1777

Fisk, L. A. 1996, J. Geophys. Res., 101, 15547

Fisk, L. A., \& Schwadron, N. A. 2001, ApJ, 560, 425

Fisk, L. A., \& Gloeckler, G. 2006, ApJ, 640, L79

Fisk, L. A., \& Gloeckler, G. 2007, Space Sci. Rev., 130, 153

Forsyth, R. J., Balogh, A., \& Smith, E. J. 2002, J. Geophys. Res. (Space Phys.), 107,1405

Gloeckler, G., \& Fisk, L. A. 2007, in Turbulence and Nonlinear Processes in Astrophysical Plasmas, ed. D. Shaikh, \& G. P. Zank, AIP Conf. Ser., 932, 264
Heerikhuisen, J., Pogorelov, N. V., Florinski, V., Zank, G. P., \& le Roux, J. A. 2008, ApJ, 682, 679

Izmodenov, V. V., Malama, Y. G., \& Ruderman, M. S. 2008, Adv. Space Res., 41,318

Kucharek, H., \& Scholer, M. 1995, J. Geophys. Res., 100, 1745

McComas, D. J., \& Schwadron, N. A. 2006, Geophys. Res. Lett., 33, 4102

McComas, D. J., Barraclough, B. L., Funsten, H. O., et al. 2000, J. Geophys. Res., 105, 10419

McComas, D. J., Elliott, H. A., Schwadron, N. A., et al. 2003, Geophys. Res. Lett., 30, 24

Müller, H.-R., Florinski, V., Heerikhuisen, J., et al. 2008, A\&A, 491, 43

Opher, M., Stone, E. C., \& Liewer, P. C. 2006, ApJ, 640, L71

Parker, E. N. 1958, ApJ, 128, 664

Pogorelov, N. V., Zank, G. P., \& Ogino, T. 2006, ApJ, 644, 1299

Pogorelov, N. V., Heerikhuisen, J., \& Zank, G. P. 2008, ApJ, 675, L41

Potgieter, M. S. 2008, Adv. Space Res., 41, 245

Richardson, J. D., Kasper, J. C., Wang, C., Belcher, J. W., \& Lazarus, A. J. 2008, Nature, 454, 63

Scherer, K., \& Ferreira, S. E. S. 2005, Ap\&SS Trans., 1, 17

Scherer, K., Ferreira, S. E. S., Potgieter, M. S., \& Fichtner, H. 2006a, in Physics of the Inner Heliosheath: Voyager Observations, Theory, and Future Prospects; 5th Annual IGPP International Astrophysics Conferenc, ed. J. Heerikhuisen, V. Florinski, G. Zank, \& P. Pogorelov, 858, 20

Scherer, K., Fichtner, H., Borrmann, T., et al. 2006b, Space Sci. Rev., 127, 327

Schwadron, N. A., \& McComas, D. J. 2003, Geophys. Res. Lett., 30, 110000

Sternal, O., Fichtner, H., \& Scherer, K. 2008, A\&A, 477, 365

Wurz, P., Galli, A., Barabash, S., \& Grigoriev, A. 2008, ApJ, to appear

Zhao, X. P., Hoeksema, J. T., \& Scherrer, P. H. 2005, J. Geophys. Res. (Space Phys.), 110, 10101 